\title{
El liderazgo democrático: una aproximación conceptual
}

\section{The democratic leadership: a conceptual approach}

Mariuxi Villalva

Universidad de Especialidades Espíritu Santo, Ecuador

Isidro Fierro

Universidad de Especialidades Espíritu Santo, Ecuador

Autor para correspondencia: mvillalva@uees.edu.ec, isfierro@uees.edu.ec

Fecha de recepción: 23 de enero de 2017 - Fecha de aceptación: 25 de Marzo de 2017

\section{Resumen}

El liderazgo democrático, también conocido como participativo, es un estilo de liderazgo que permite crear mayor compromiso con la organización pues se centra en los subordinados involucrándolos en la toma de decisiones, delegando autoridad y utilizando la retroalimentación como un medio para dirigir y corregir errores. Se procedió hacer una búsqueda bibliográfica en las bases de datos sobre el concepto y características del liderazgo y liderazgo democrático. A partir del análisis de diversos puntos de vista enfocados a éste tema se concluye que el liderazgo democrático facilita la integración, fomenta el trabajo en equipo, permite la participación activa en la toma de decisiones mediante el dialogo y por tanto incrementa la satisfacción en el trabajo, el desarrollo profesional y mejora la calidad de las decisiones para alcanzar los objetivos propuestos.

Palabras claves: liderazgo; liderazgo democrático; toma de decisiones; trabajo en equipo

\begin{abstract}
Democratic leadership, also known as participatory, is a leadership style that allows creating a greater commitment with the organization as it focuses in the subordinates involving them in the decision making, delegating authority and using feedback as a means to direct and correct errors. Proceeded to do a bibliographical research in the databases on the concept and characteristics of leadership and democratic leadership. From the analysis of different points of view this issue is concluded that democratic leadership facilities the integration, foments team works, allows active participation in making decisions, through dialogue and therefore increases job satisfaction, professional development and improves the quality of decisions to achieve the proposed objectives.
\end{abstract}

Key words: leadership; democratic leadership; decision making; team work 


\section{Introducción}

Desde el inicio de los tiempos, se han destacado personas capaces de inspirar, influir y guiar a grupos de personas para lograr un propósito en común. Estas personas se las conoce como líderes. Echart y Rodríguez (2014) define a un líder como una persona que inspira confianza y seguridad a un grupo de personas quienes por decisión unánime lo escogen para que esté al frente de un equipo, organización o sociedad y mediante sus decisiones y trabajo en conjunto poder cumplir con los objetivos trazados.

En la actualidad las organizaciones se enfrentan a niveles de competencia cada vez más rigurosos. Según Lupano y Castro (2008) el liderazgo es indispensable para guiar a las organizaciones y los recursos humanos hacia objetivos estratégicos; también es vital ya que da la posibilidad de contar con los mejores recursos, buena planificación, control y supervisión, pero si no se tiene un líder apropiado es difícil que sobreviva la organización (Noriega, 2008). Por tanto, el tipo de liderazgo que tengan los gerentes es un factor clave para el éxito de las organizaciones (Fierro, 2012).

Existen diferentes tipos o estilos de liderazgo que se basan específicamente en el comportamiento del líder, su estilo conductual, es decir en lo que hace y cómo lo hace (Palomino, 2009). El estilo de liderazgo influye sobre la conducta de los individuos en la empresa (Shamir, House y Arthur, 1993), así como en los procesos de trabajo grupal, el clima social y los resultados (Evkall y Ryhammar, 1997; Kahai y Sosik, 1997).

Lewin (1951) definió una de las primeras explicaciones de los estilos de liderazgo argumentando que éstas surgen del uso que los dirigentes dan a la autoridad que poseen. Cabe destacar un estilo liderazgo denominado "liderazgo democrático o participativo" el cual se centra en los subordinados, es decir los involucra en la toma de decisiones, delega autoridad y emplea la retroalimentación como una oportunidad para dirigir, sin dejar a un lado su opinión.

Este artículo tiene como objetivo mostrar la definiciones, beneficios y características del liderazgo democrático mediante una aproximación conceptual tomando como referencia el punto de vista de diversos autores expertos en temas de liderazgo tales como Warrick, Bass, Adams y Yoder, que permitirán demostrar la viabilidad de este modelo de liderazgo a nivel general.

Para comprender de una mejor manera sobre el liderazgo, es importante revisar sus orígenes, sus primeros planteamientos teóricos y sus características los cuales serán tratados en la revisión conceptual.

\section{Revisión conceptual}

\section{Liderazgo: Antecedentes}

El liderazgo ha estado presente en diversos personajes de la sociedad, tanto históricos, religiosos, militares y políticos logrando mover tanto a grupos pequeños como a grandes masas. 
Desde los tiempos de la Grecia antigua la conceptualización más común ha sido enfocarse en un sólo líder y sus características. La teoría de rasgos (Stogdill, 1948; Ghiselli 1970) plantea que los líderes nacen y no se hacen; cuentan con características innatas de inteligencia, intuición, capacidad, previsión, integridad, que les permiten enfrentar temas de urgencia, y la pronta y adecuada gestión de procesos e imprevistos.

Posteriormente, se concibe que el liderazgo no es una cualidad innata, los líderes aprenden a serlo. Según Moss (1992) el liderazgo es una habilidad del comportamiento, por lo que se puede aprender a desarrollar por sí mismo. Es un proceso que se adquiere, que implica destrezas y habilidades. (Álvarez, 1998). Así el aprendizaje garantiza la obtención de numerosas destrezas, que el líder podrá poner en práctica en inimaginables situaciones, lo que, en cierto modo, garantizará una actuación más acertada.

Es necesario analizar el liderazgo tomando como referencia el punto de vista de autores especializados en ello, por tal razón se expondrán los conceptos que han establecido según sus investigaciones.

\section{Liderazgo: Definición}

El liderazgo no cuenta con un concepto fijo debido a que diversos autores lo designan de acuerdo a sus experiencias y estudios realizados (Reyes, 2014).

Fiedler (1961), citado por Kreitner y Kinicki (1997), considera que el liderazgo es un «hecho subjetivo» que determina el poder de un grupo mediante las relaciones entre el líder y los demás miembros del grupo.

Según Korman, Greenhaus y Badin (1977) el liderazgo es generar que un individuo A persuada a B para que realice lo que A quiere. Sin embargo, se han presentado otras disposiciones para que se considere que A influye en B en algo que es bueno para ambos.

Buono y Bowditch (1989) y Rost (1991) definen al liderazgo como el esfuerzo que se realiza para influir en el comportamiento de los demás, o para ordenar que se logren los objetivos organizacionales, individuales o personales.

Para Robbins (1999), el liderazgo es la capacidad de influir en un grupo para alcanzar las metas propuestas. Es decir, considera que el liderazgo es la influencia interpersonal que se ejerce por medio de un proceso de comunicación humana y que se analiza en función de las relaciones existentes entre las personas dentro de cierta estructura social.

Según las investigaciones de Lussier y Achua (2002) se refieren al liderazgo como un "proceso en el cual influyen líderes sobre seguidores y viceversa, para lograr los objetivos de una organización a través del cambio".

Hoy en día, en este mundo globalizado y complejo, el líder es visto como un facilitador de cambios emergentes dentro de la organización, que debe tener la capacidad de guiar la cooperación en la realización de las tareas y en la consecución de los objetivos (Contreras \& 
Barbosa, 2013; Schneider, 2002). Por tanto, el líder debe poseer la habilidad de gestionar el capital humano, influenciar las fuerzas del entorno e integrarlas a la organización creando valor.

\section{Liderazgo: Características}

Un líder debe poseer la capacidad cognitiva, social y comportamental para contribuir al desarrollo de equipos altamente efectivos (Uribe, Molina, Contreras, Barbosa \& Espinosa, 2013). Desde este enfoque, el liderazgo implica el desarrollo de habilidades, fomentar la participación, la creatividad y el crecimiento personal de todos los miembros de la organización (McCollum, 1999; Perdomo \& Prieto, 2009).

Kouzes y Posner (1997) hacen referencia a cinco comportamientos que los líderes altamente efectivos expresan constantemente:

- Desafiar los procesos: implica el desafío al cambio, al cuestionamiento del status quo, a correr riesgos y aprender a generar innovaciones en los procesos que lleva la organización.

- Inspirar una visión compartida: con qué frecuencia el líder comparte la visión con sus seguidores y qué tan comprometido está el líder con la visión de la organización.

- Facultar a otros para actuar: es la delegación y empoderamiento que el líder hace o da a sus seguidores, a la promoción que el líder hace de la colaboración mutua entre los seguidores y que tanto permite el líder que sus seguidores realicen tareas importantes.

- Servir de modelo: hace referencia al líder como ejemplo de comportamiento, a lo que demuestra el líder de cómo deben comportarse los seguidores, cómo el líder manifiesta sus valores y la coherencia entre lo que dice y lo que hace el líder.

- Brindar aliento: Esta última práctica hace referencia a la manera en que el líder reconoce los logros tanto individuales como grupales de sus seguidores.

Luego de revisar el constructo del liderazgo se procederá a tratar la base de este artículo la cual es el liderazgo democrático por lo que se iniciará con las respectivas definiciones tomando en cuenta los puntos de vista de autores que han desarrollado sus investigaciones en torno a ello.

\section{Liderazgo democrático: Definición}

Lewin (1951) definió una de las primeras explicaciones de los estilos de liderazgo basándose específicamente en el comportamiento del líder. El estilo de liderazgo democrático también llamado liderazgo participativo describe a un líder que involucra a los subordinados en la toma de decisiones, delega autoridad, fomenta la decisión de métodos de trabajo y sus metas y emplea la retroalimentación como una oportunidad para dirigir.

Dentro de este enfoque la teoría del liderazgo participativo de Vroom y Yetton, (1973) propone que el liderazgo es una conducta que se asocia a los grupos y no solo a los individuos, señala que todo individuo en una organización está capacitado para contribuir a las metas colectivas. La intención de Vroom y Yetton no fue proponer una gran teoría del liderazgo, sino más bien centrar su interés en la manera en que los gerentes comparten el poder, ofrecen 
participación e influyen sobre sus subordinados; es decir, la toma de decisiones. En este modelo también se apoyaron en las investigaciones realizadas por Coch y French (1948), French, Israel y As (1960) y Morse y Reimer (1956) respecto a la relación existente entre la participación de los empleados en la toma de decisiones y la eficacia de ésta, y el uso efectivo de la participación en función de las circunstancias de la situación.

Para Warrick (1981) un líder democrático realiza énfasis en el desempeño y las personas. Este tipo de líder asume que la mayoría de la gente es honesta, de alta confianza, y trabajará duro para lograr objetivos significativos y trabajo desafiante. Se esfuerza por ser organizado, haciendo el trabajo mediante la motivación y la gestión de las personas y grupos para que usen su potencial para cumplir los objetivos de la organización, así como sus propios objetivos personales.

Adams y Yoder (1985) describe al líder democrático como un instrumento para promover el bienestar del grupo, está abierto a las opiniones de sus seguidores, permite participación en la toma de decisiones y considera que los seguidores se encuentran motivados para hacer lo correcto. El poder del líder reside en la interacción con los subordinados, dado que el grupo le otorga el poder y el líder como respuesta asume la responsabilidad de dirigirlos sin llegar a centralizar el poder. Este tipo de líder comparte el poder (Bass, 2008). La creación de este tipo de liderazgo implica la discusión de todas las políticas y decisiones de la organización que finalmente son tomadas por el grupo con la guía y participación activa del líder en las actividades.

Robbins y Coulter (1999) explica que en el Liderazgo Democrático-Participativo el gerente sí delega su autoridad en otros, comparte el control y la toma de decisiones la hace en conjunto, sin dejar de supervisar el cumplimiento de los objetivos. Es un poco permisivo y deja que los otros integrantes del grupo también evalúen su propio desempeño, sin dejar a un lado su opinión.

\section{Características del líder Democrático}

Para Robbins y Coulter (1999) las características de un líder democrático son: 1) Delega autoridad y comparte el control, 2) Toma las decisiones en conjunto y delega funciones, 3) Otorga confianza al grupo y se apoya en los seguidores para dictar las pautas y 4) Emplea el feedback y corrige errores para incentivar.

Según Gonos y Gallo (2013) un liderazgo democrático se determina por las siguientes características: 1) Los subordinados están involucrados en el proceso de toma de decisiones, 2) Los gerentes están constantemente tratando de obtener el consentimiento de los subordinados antes de implementar los cambios, 3) Los gerentes y subordinados se reúnen para discutir los problemas relacionados con el trabajo, 4) Los gerentes informan al grupo sobre temas relevantes, el progreso, relaciones con otros grupos y así sucesivamente, 5) Los líderes explican sus intenciones y la situación actual, y 6) Los gerentes toman tiempo para escuchar a los miembros del grupo y se interesan por los sentimientos y las ideas de los miembros del grupo. 
También es muy importante que el liderazgo participativo cuente con: 1) Tratamiento de conflictos, dado que el consenso es un factor indispensable en este tipo de liderazgo, 2) Alta motivación, pedir opiniones y aceptar sugerencias permite que el equipo se sienta parte del proceso, 3) Esfuerzo común, el esfuerzo que aporta cada miembro del grupo forma parte de una cadena de acciones para alcanzar los objetivos compartidos y 4) Fomentar la responsabilidad es vital para entender el rol que desempeñan dentro del conjunto y guiar sus esfuerzos. (Harvard Deusto Business Review, 2017).

\section{Conclusión}

Un líder es democrático cuando fomenta el trabajo en equipo, el diálogo, los acuerdos por consenso, el cumplimiento de los deberes de todos los miembros del grupo, no actúa de manera individual, sino que lo hace con el equipo y en su representación.

La participación, como estilo de dirección, permite tener mayor compromiso de los trabajadores con la organización, generando mayor motivación para alcanzar los objetivos planteados y facilitar la integración del factor humano en la organización por consiguiente incrementa la satisfacción en el trabajo, el desarrollo profesional, la actitud positiva de los trabajadores hacia sus dirigentes y mejora la calidad de las decisiones para alcanzar los objetivos propuestos.

Cuando se permite la participación en la toma de decisiones se está llevando a cabo un entrenamiento para mejorar la habilidad en la toma de decisiones futuras, no solo se limita en ejecutarlas. Al formar parte en el proceso de toma de decisiones los miembros del equipo pueden desarrollar sus relaciones interpersonales y el sentimiento de pertenencia en el grupo. Otro beneficio relacionado con el desarrollo personal mediante la participación es la autodirección, que permite el incremento de la autoconfianza de los participantes del equipo.

Podemos resumir que el liderazgo democrático se caracteriza en promover la participación de todo el grupo de trabajo, mediante el dialogo, entusiasmo y compromiso. El líder debe tener en cuenta todas las opiniones de su grupo de trabajo, facilitar la comunicación, delegar tareas y confiar en la capacidad de su grupo, fomentar su participación sin marginación alguna, enfocarse en un objetivo en común y estimular el sentido de pertenencia de la empresa. Evidentemente que el liderazgo democrático es positivo, pero habrá situaciones en las que sea más adecuado utilizar otros estilos de liderazgo para llegar a decisiones de calidad.

\section{Bibliografía}

Adams, J. y Yoder, J. D. (1985). Effective leadership for women and men. Norwood, NJ: Ablex.

Álvarez, M. (1998). El liderazgo de la calidad total. Madrid: Editorial Escuela Español.

Bass, B. M. (2008). The Bass handbook of leadership. Theory, research \& managerial applications (4.a ed.). New York: Free Press.

Buono, Anthony F.; Bowditch, James L. The Human Side of Mergers and Acquisitions: managing collisions between people, cultures, and organizations, 1989. 
Coch, Lester and FRENCH Jr., John R. P. Overcoming resistance to change. In: Human Relations. November, 1948. vol 1, no. 4, p. 512-532

Contreras, F. \& Barbosa, D. (2013). Del liderazgo transaccional al liderazgo transformacional: Implicaciones para el cambio organizacional. En Prensa, Revista Virtual Universidad Católica del Norte.

Echart, P., \& Rodríguez, J. (2014). Un liderazgo transformacional y ejemplar: la imagen de Nelson Mandela en Invictus. Empresa y Humanismo, 7-22.

Evkall, Gãfâfã; Ryhammar, Lars. Leadership style, social climate and organizational outcomes: A study of a Swedish University College. In: Creativity and Innovation Management, 1998, vol. 7, no 3, p. 126-130.

Fierro, I. (2012). El Rol Del Liderazgo Estratégico En Las Organizaciones. Saber, Ciencia y Libertas, $7(1)$.

French, John; Israel, Joachim; AS, Dagfinn. An experimental study on participation in a Norwegian factory. In: Human Relations, 1960. Vol 13, p. 3-19.

Ghiselli, E. (1970). Psicología industrial, su aplicación al personal de la empresa. La Habana: Revolucionaria.

Gonos, J. y Gallo, P. (2013). Model for leadership style evaluation. Management, 18(2), 157-168.

Harvard Deusto Business Review y EAE Business School (2017). Características principales del liderazgo participativo. Recuperado el 19 Febrero del 2017, de http://retosdirectivos.eae.es/caracteristicas-principales-del-liderazgo-participativo/

Kahai, Solen.; SOSIK, Jennifer. Effects of leadership style and follower's cultural orientation on performance in group and individual task conditions. In: Personnel Psychology, 1997, vol. 50, no 1 , p. 121- 147.

Korman, Abraham K.; Greenhaus, Jeffrey H.; Badin, Irwin J. Personnel attitudes and motivation. In: Annual Review of Psychology, 1977, vol. 28, no 1, p. 175-196.

Kouzes, J. \& Posner, B. (1997).The leadership challenge. San Francisco, USA: Jossey-Bass Inc.

Kreitner, R. y Kinicki, A. (1997). Comportamiento de las organizaciones. Madrid: McGraw Hill.

Lewin, Kurt. Field theory in social science. 1951.

Lupano, M., y Castro, A. (2008). Estudios sobre el liderazgo: Teorías y evaluación. Psicodebate 6: $\begin{array}{lllll}\text { Psicología, } & \text { Cultura } & \text { y } & \text { Sociedad, } & \text { 107-122. }\end{array}$ http://www.palermo.edu/cienciassociales/publicaciones/pdf/Psico6/6Psico\%2008.pdf

Lussier, Robert, Achua Christopher. Liderazgo. Teoría, Aplicación y Desarrollo de habilidades (2a. Edición). México: Thomson Learning, 2002. 
McCollum, B. (1999). Leadership development and self-development: An empirical study. Career Development International, 4 (3), 149-156.

Morse, Nancy; Reimer, Everett. The experimental change of a major organizational variable. In: The Journal of Abnormal and Social Psychology, 1956, vol. 52, no 1, p. 120.

Moss, G. (1992). Ser líder. Manual para conseguir objetivos. Valencia: Jumerca.

Noriega, M. (2008). La importancia del liderazgo en las organizaciones. Temas de Ciencia y Tecnología, 12(36), 25-29. http://www.elfinancierocr.com/gerencia/ biblioteca/GuadalupeNoriega-Universidad-Tecnologica-ixteca_ELFFIL20140425_0008.pdf

Palomino, Pablo Ruiz. Últimas tendencias en el estudio sobre liderazgo: Revisión de la literatura. En: Documentos de Trabajo. Seminario Permanente de Ciencias Sociales, 2009, no 5, p. 1-16.

Perdomo, Y. \& Prieto, R. (2009). El liderazgo como herramienta de competitividad para la gerencia del servicio. Revista Electrónica de Estudios Temáticos, 6 (2). 20-35. Recuperado el 22 de junio de 2013, de http://www.publicaciones.urbe.edu/index.php/cicag/article/ viewArticle/469

Reyes, R. (2014). Liderazgo en el Siglo XXI. Pachuca: Centro Universitario Siglo XXI.

Robbins, S. y Coulter, M. (1999) Administración. Quinta edición. Prentice Hall. México.

Robbins, Stheven. Comportamiento organizacional (8ª edición). México: Prentice Hall, 1999.

Rost, Joseph C. Leadership for the twenty-first century. 1991.

Schneider, M. (2002). A stakeholder model of organizational leadership. Organization Science, 13, 209-220.

Shamir, Boas; Arthur, Michael B.; House, Robert J. The rhetoric of charismatic leadership: A theoretical extension, a case study, and implications for research. In: The Leadership Quarterly, 1994, vol. 5, no 1, p. 25-42.

Stogdill, R. M. (1948). Personal factors associated with leadership: A survey of the literature. Journal of Psychology, 25, 35-71.

Uribe, A., Molina, J., Contreras, F., Barbosa, D. \& Espinosa, J. (2013). Liderar Equipos de alto desempeño: Un gran reto para las organizaciones actuales. En prensa Revista Universidad y Empresa U\&E.

Vroom, V. y Yetton, P. (1973). Leadership and decision making. Pittsburgh: University of Pittsburgh Press.

Warrick, D. (1981). Leadership styles and their consequences. Journal of Experiential Learning and Simulation, 3-4, 155-172. 